\title{
Nausea, CTCAE 5.0
}

National Cancer Institute

\section{Source}

National Cancer Institute. Nausea, CTCAE 5.0. NCI Thesaurus. Code C146764.

A disorder characterized by a queasy sensation and/or the urge to vomit. 